\title{
Gesundheit wird immer teurer - nur wir Ärzte nicht
}

Chefredakteur Dr. med. Dirk Einecke

\section{Die Gesundheits- Gesamtrechnung}

Im Jahr 2005 sind 239,4 Milliarden Euro (10,7\% des Bruttoinlandsprodukts) für die Gesundheit ausgegeben worden. Dies entspricht einem Plus von 5,6 Milliarden Euro gegenüber 2004. Jeweils $\mathbf{2 7} \%$ der

Ausgaben entfielen auf Waren und auf ärztliche Leistungen. Damit wurden für ärztliche Leistungen nur $0,5 \%$ mehr aufgewendet als im Vorjahr.

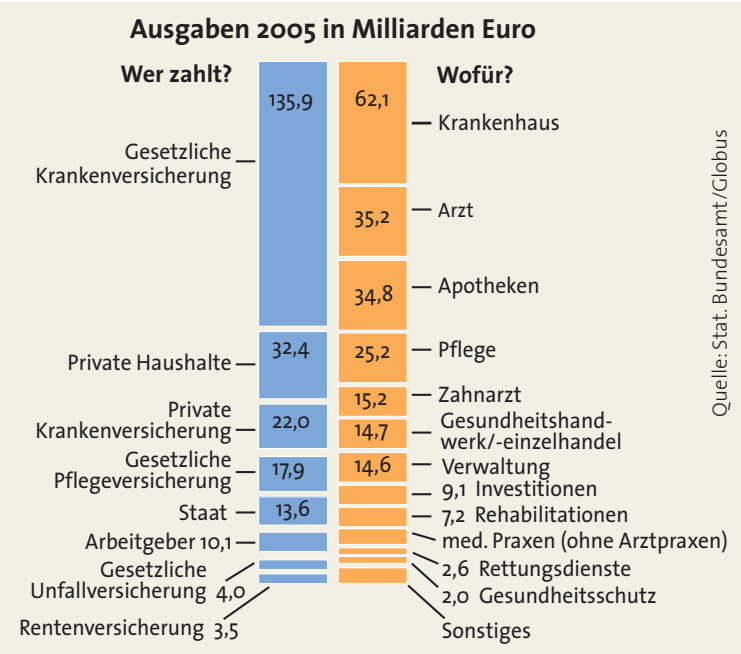

HYPOGLYKÄMIEN BEI TYP-1-DIABETIKERN

\section{Keine Langzeitfolgen für die geistige Leistungsfähigkeit}

Eine strenge Blutzuckerkontrolle bewahrt Typ-1-Diabetiker besser vor mikro- und makrovaskulären Folgekomplikationen als eine laschere Stoffwechseleinstellung. Der Preis dafür ist allerdings ein erhöhtes Risiko für Hypoglykämien. Zumindest im Hinblick auf die Langzeitfolgen dieser Episoden kann aber Entwarnung gegeben werden. Selbst schwere Unterzuckerun- gen ziehen keine kognitiven Beeinträchtigungen nach sich. Das zeigt eine Studie mit mehr als 1100 Typ-1-Diabetikern, die über einen Zeitraum von 18 Jahren beobachtet wurden und von denen $40 \%$ mindestens einmal hypoglykämiebedingt ein Koma oder einen Krampfanfall erlebt hatten.

N Engl J Med 2007;356:1842-52

\section{SCHADSTOFF FÜRS GEHIRN}

\section{Passivrauchen erhöht Demenzrisiko}

Wer jahrzehntelang unfreiwillig Zigarettenqualm einatmen muss, hat zu allen anderen Unannehmlichkeiten auch noch mit einem erhöhten Demenzrisiko zu rechnen. Dies wurde im Rahmen der Cardiovascular Health Study mit 3602 Teilnehmern über 65 Jahren festgestellt. Diejenigen Probanden, die im Laufe ihres Lebens mindestens 30 Jahre lang Passivrau- cher waren, hatten ein um ca. 30\% höheres Risiko, eine Demenz zu entwickeln, als Nichtraucher, die keinem regelmäßigen Passivrauch ausgesetzt waren. Passivraucher, die zudem bereits Karotisstenosen aufwiesen, hatten ein mehr als zweieinhalbfaches Demenzrisiko.

59. Jahrestagung der American Academy of Neurology, Boston, 28.4.-5.5.2007

\section{Zitat der Woche}

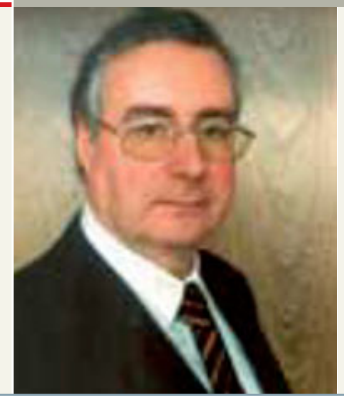

„Man kann doch eine Gesundheitsministerin nicht zu einer Gruppe von Geiselnehmern einladen. Das wäre zu gefährlich."

Dr. Joachim Calles, Delegierter des 63 . Bayerischen Ärztetags am 28. April 2007 in Nürnberg, zum Antrag, Bundesgesundheitsministerin Ulla Schmidt wegen des Vorwurfs der "Geiselnahme von Patienten“ durch Ärzte vom 110. Deutschen Ärztetag wieder auszuladen.

\section{JE MEHR, DESTO SCHLIMMER}

\section{Alkohol lässt das Gehirn schrumpfen}

Starke Trinker haben ein kleineres Hirnvolumen als Abstinenzler. Dieser Zusammenhang wurde bei 1839 Teilnehmern der Framingham-Offspring-Studie mittels MRT-Untersuchung objektiviert. Das Hirnvolumen war umso niedriger, je mehr Alkohol regelmäßig konsumiert wurde. Bei Frauen um die 70 war der negative Zusammenhang am stärksten ausgeprägt.

59. Jahrestagung der American Academy of Neurology, 28.4.-5.5.2007

\section{ELTERN LEIDEN AN ALLERGIE}

\section{Keine Katze fürs Kind}

Mit einer Katze im Haus steigt bei Kindern bis zu zwei Jahren das Risiko einer allergischen Sensibilisierung. Bei Sechsjährigen ist - wahrscheinlich aufgrund ihres größeren Aktionsradius - dieser Effekt aber nicht mehr feststellbar. Bei beiden Altersgruppen wird bereits durch wiederholten Kontakt mit Katzenhaaren eine entsprechende Sensibilisierung begünstigt. Zu diesem Ergebnis kommt eine deutsche Studie, in die 2166 Kinder einbezogen waren. Insgesamt fanden die Forscher bei 1,3\% der Zweijährigen und bei $5 \%$ der Sechsjährigen spezifische IgEAntikörper gegen Katzenallergene. Vor allem Eltern, die selbst an Allergien leiden, so die Empfehlung der Autoren, sollten keine Katze halten und ihre Kinder auch sonst von Katzen fernhalten.

J Allergy Clin Immunol 2007;119:1148-55

\section{BEI ALTEN MENSCHEN GILT}

\section{Snacks sind gesund}

Regelmäßig zwischen den Mahlzeiten zu essen, kann eine übermäßige Kalorienaufnahme begünstigen. Das gilt jedoch nicht bei über 70-Jährigen: Weil hier Malnutrition und nicht Adipositas das wichtigste Ernährungsproblem darstellt, sind Zwischenmahlzeiten sogar zu begrüßen. Eine Studie aus den USA zeigt, dass alte Menschen, die regelmäßig kleine Snacks zu sich nehmen, ihren Kalorienbedarf besser decken als „Nicht-Snacker“. American Dietetic Association, Mai 2007 